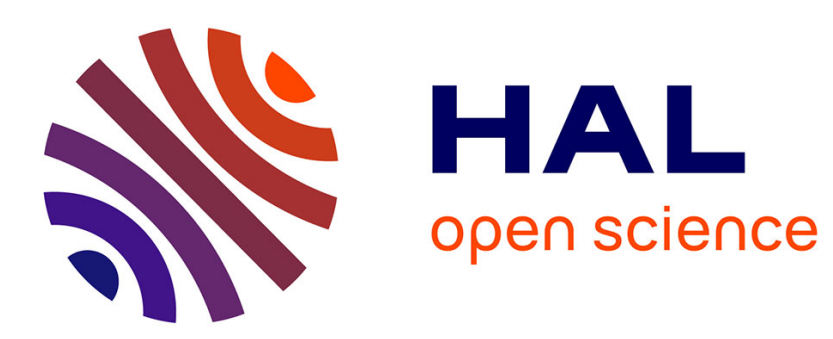

\title{
Distributed approximation of open-channel flow routing accounting for backwater effects
}

\author{
Simon Munier, Xavier Litrico, Gilles Belaud, P.-O. Malaterre
}

\section{To cite this version:}

Simon Munier, Xavier Litrico, Gilles Belaud, P.-O. Malaterre. Distributed approximation of openchannel flow routing accounting for backwater effects. Advances in Water Resources, 2008, 31 (12), pp.1590-1602. 10.1016/j.advwatres.2008.07.007 . hal-00682721

\section{HAL Id: hal-00682721 \\ https://hal.science/hal-00682721}

Submitted on 26 Mar 2012

HAL is a multi-disciplinary open access archive for the deposit and dissemination of scientific research documents, whether they are published or not. The documents may come from teaching and research institutions in France or abroad, or from public or private research centers.
L'archive ouverte pluridisciplinaire HAL, est destinée au dépôt et à la diffusion de documents scientifiques de niveau recherche, publiés ou non, émanant des établissements d'enseignement et de recherche français ou étrangers, des laboratoires publics ou privés. 


\title{
Distributed approximation of open-channel flow routing accounting for backwater effects
}

\author{
Simon Munier ${ }^{\mathrm{a}}$ Xavier Litrico ${ }^{\mathrm{a}}$ Gilles Belaud $^{\mathrm{b}}$ \\ Pierre-Olivier Malaterre ${ }^{\text {a }}$

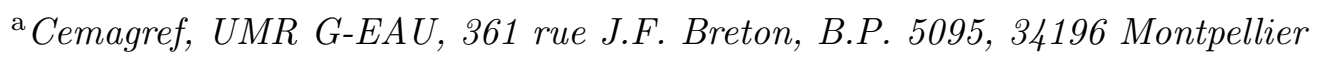 \\ Cedex 5, France \\ ${ }^{\mathrm{b}}$ IRD, UMR G-EAU, Maison des Sciences de l'Eau, 300 Avenue Emile Jeanbrau, \\ 34095 Montpellier Cedex 5, France
}

\begin{abstract}
In this article, we propose a new model, called LBLR for Linear Backwater Lagand-Route, which approximates the Saint-Venant equations linearized around a non-uniform flow in a finite channel (with a downstream boundary condition). A classical frequency approach is used to build the distributed Saint-Venant transfer function providing the discharge at any point in the channel in the Laplace domain with respect to the upstream discharge. The moment matching method is used to match a second-order-with-delay model on the theoretical distributed Saint-Venant transfer function. Model parameters are then expressed analytically as functions of the pool characteristics. The proposed model efficiently accounts for the effects of downstream boundary condition on the channel dynamics.
\end{abstract}

Key words: Open-channel flow routing, Saint-Venant equations, frequency response, Laplace transform

\section{Introduction}

Water resources are renewable but in limited supply. In a context of multiple needs, such as irrigation or domestic water supply, this resource has to

Email addresses: simon.munier@cemagref.fr (Simon Munier), xavier.litrico@cemagref.fr (Xavier Litrico), belaud@msem.univ-montp2.fr (Gilles Belaud), pierre-olivier.malaterre@cemagref.fr (Pierre-Olivier Malaterre). 
be collected, shared and then distributed using water transport systems, such as rivers and/or canals. Water managers control flows in such open-channels using hydraulic structures (dams, weirs, gates). The water distribution efficiency can be greatly improved by operating these structures using automatic tools. This requires models that are able to represent open-channel flow routing with a desired accuracy. Nowadays, many systems are controlled using linear controllers based on linear models since many methodologies and tools are well-known and validated for such systems. Linear approaches have also advantages in terms of simplicity, rapidity and robustness $([11,13,17])$. Several authors used numerical schemes to obtain such linear models from the Saint-Venant equations $([1,10,21])$. But they are usually high order models. Alternative approaches could be preferred to obtain lower order models with coefficients expressed analytically. This justifies the interest in improving such flow routing models.

Modeling of flow routing along a river stretch or a canal pool has been the subject of numerous articles since the 1950's. A comprehensive review of approximate flow routing methods has been presented in [28]. Different linear models have been developed for flow routing simulation purposes (e.g. [8,26]). Most of them are based on analysis of the linearized Saint-Venant equations around a reference flow. To greatly simplify the equations, the downstream boundary condition is usually neglected by considering a semi-infinite channel (see e.g. [8]), leading to a uniform reference flow. However, a downstream boundary condition imposed by a hydraulic structure, such as a weir, has two different effects on the flow: it modifies the water depth, causing a backwater curve, and it enforces a local coupling, called feedback, between the discharge and water depth. But, even in a linear framework (i.e. for small variations), neglecting these two effects sometimes leads to large under- or over-estimation of some parameters such as the response time, peak time or the level of attenuation. The effects induced on the flow dynamics by the downstream boundary condition have been analyzed based on numerical simulations in [25]. Some analysis of the backwater effects has also been brought in [7] and [27], and has underlined the fact that the downstream boundary condition can sometimes provide significant modifications of the flow dynamics.

In this article, a new three-parameter model, called LBLR for Linear Backwater Lag and Route, is derived and accounts for the effects of the downstream boundary condition. It takes the feedback and the backwater effects into account separately, and provides the discharge at any point in the channel with respect to the discharge at the upstream end. The three parameters are expressed analytically depending on the pool characteristics (length, geometry, roughness, reference flow), which provides a quick and accurate calculation of these parameters.

Our approach is based on the frequency domain representation of linearized 
Saint-Venant equations, which generates transfer functions in the Laplace domain. In this framework, the introduction of a downstream boundary condition is equivalent to a local feedback between the discharge and the water level deviations. A moment matching method is then used to compute the parameters of a first- or a second-order-with-delay model that approximates the system in low frequencies. The obtained model is first computed in the uniform case, then extended to the non-uniform flow. In the last section, the model is validated on a sample canal under different downstream boundary conditions.

\section{Methodology based on a frequency approach}

\subsection{General methodology}

We consider the full one-dimensional Saint-Venant equations for a prismatic channel of length $X$ linearized around a reference steady state regime, possibly non-uniform (see Fig. 1).

These equations are rewritten in the Laplace domain, which leads to the SaintVenant transfer matrix linking the discharge and the water depth at any point in the channel to the two boundary conditions: downstream and upstream discharges.

Since the downstream boundary condition introduces a local coupling between the downstream discharge and the downstream water depth (feedback), this relation can be combined with the Saint-Venant transfer matrix, reducing the number of boundary conditions to only one: the upstream discharge. This feedback relation is linearized, prior to its Laplace transformation. Coupling the obtained relation with the Saint-Venant transfer matrix leads to the desired transfer function, linking, in the Laplace domain, the upstream discharge to the discharge at any point in the channel.

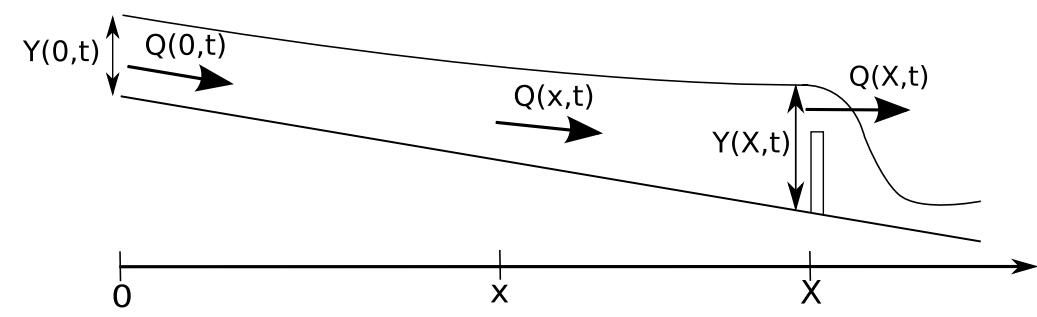

Fig. 1. General scheme of the considered channel. 


\subsection{Linearized Saint-Venant equations}

We consider a stationary regime and small variations around it. The discharge of the reference flow $Q$ is assumed to be constant along the channel, whereas the water depth can vary. The following notations are used: $x(\mathrm{~m})$ is the abscissa along the channel, $S_{b}$ the bed slope and $g$ the gravitational acceleration $\left(\mathrm{ms}^{-2}\right)$. The following variables represent the reference flow: $A(x)$ the wetted area $\left(\mathrm{m}^{2}\right), P(x)$ the wetted perimeter $(\mathrm{m}), Q$ the discharge $\left(\mathrm{m}^{3} / \mathrm{s}\right)$ through section $A(x), Y(x)$ the water depth $(\mathrm{m}), S_{f}(x)$ the friction slope, $V(x)=Q / A(x)$ the mean flow velocity $\left(\mathrm{ms}^{-1}\right)$ and $T(x)$ the top width $(\mathrm{m})$, $F(x)=V(x) / \mathcal{C}(x)$ the Froude number with $\mathcal{C}(x)=\sqrt{g A(x) / T(x)}$ the wave celerity $\left(\mathrm{ms}^{-1}\right)$. Throughout the article, the flow is assumed to be subcritical (i.e., $F(x)<1$ ).

The friction slope $S_{f}$ is modeled using the Manning formula (see [6]):

$$
S_{f}(x)=\frac{Q^{2} n^{2}}{A(x)^{2} R(x)^{4 / 3}}
$$

with $n$ the Manning coefficient $\left(\mathrm{sm}^{-1 / 3}\right)$ and $R(x)$ the hydraulic radius $(\mathrm{m})$, defined by $R(x)=A(x) / P(x)$.

Let us denote $q(x, t)$ and $y(x, t)$ the variations in discharge and water depth at abscissa $x$ and time $t$, compared to the reference steady regime.

The linearized Saint-Venant equations are given by (see [14] for details):

$$
\begin{aligned}
T \frac{\partial y}{\partial t}+\frac{\partial q}{\partial x} & =0 \\
\frac{\partial q}{\partial t}+2 V \frac{\partial q}{\partial x}-\mu q+\left(\mathcal{C}^{2}-V^{2}\right) T \frac{\partial y}{\partial x}-\nu y & =0
\end{aligned}
$$

where the dependency on $x$ and $t$ is omitted for clarity purposes.

In the general non-uniform case, parameters $\mu$ and $\nu$, which are functions of $x$, are defined by the following equations:

$$
\begin{aligned}
& \nu=V^{2} \frac{d T}{d x}+g T\left[(1+\kappa) S_{b}-\left(1+\kappa-(\kappa-2) F^{2}\right) \frac{d Y}{d x}\right] \\
& \mu=-\frac{2 g}{V}\left(S_{b}-\frac{d Y}{d x}\right)
\end{aligned}
$$

with $\kappa=7 / 3-4 A /(3 T P)(\partial P / \partial Y)$. 
The two boundary conditions are the upstream discharge denoted $q_{0}(t)=$ $q(0, t)$ and the downstream discharge denoted $q_{X}(t)=q(X, t)$.

\subsection{Frequency approach}

The purpose of the article is to establish a transfer function $T F(x, s)$ linking the upstream discharge to the discharge at any point in the channel.

$$
q_{x}(s)=T F(x, s) q_{0}(s)
$$

For this, the Laplace transform is applied to the Saint-Venant equations, leading to an ordinary differential equation in the space variable $x$ and parameterized by the Laplace variable $s$. The integration of this equation leads to a transfer matrix $\Gamma(x, s)$, called the transition matrix, and gives the discharge $q(x, s)$ and the water depth $y(x, s)$ at any location with respect to the upstream discharge $q_{0}(s)$ and water depth $y_{0}(s)$. This matrix is then coupled with a feedback relation introduced with the downstream boundary condition, which leads to an analytical expression of the transfer function $T F(x, s)$.

\subsection{Laplace transform}

The Laplace transform $\mathcal{L}$ of a function $f(t)$ is defined as follows:

$$
\mathcal{L}\{f\}(s)=\int_{0^{-}}^{+\infty} e^{-s t} f(t) d t
$$

where $s$ is the Laplace variable.

The following property is used to derive the Saint-Venant equations in the Laplace domain:

$$
\mathcal{L}\left\{\frac{d f}{d t}\right\}(s)=s \mathcal{L}\{f\}(s)-f\left(0^{-}\right)
$$

The boundary $0^{-}$is chosen to prevent troubles at the origin, especially when the function $f$ is discontinuous (see [19]).

Taking some liberty with notation, we denote $f(s)=\mathcal{L}\{f\}(s)$. The possible ambiguity with $f(t)$ will be clarified contextually. 


\subsection{Saint-Venant transfer matrix}

Applying Laplace transform to the linear partial differential Eqs. (2) and (3) results in a system of Ordinary Differential Equations (ODE) in the variable $x$, parameterized by the Laplace variable $s$.

The integration of this ODE leads to the transition matrix $\Gamma(x, s)$ linking the discharge $q$ and the water depth $y$ at any point $x$ in the canal pool to the upstream discharge and water depth, $q_{0}$ and $y_{0}$ respectively (see appendix A for details):

$$
\left(\begin{array}{l}
q(x, s) \\
y(x, s)
\end{array}\right)=\Gamma(x, s)\left(\begin{array}{l}
q_{0}(s) \\
y_{0}(s)
\end{array}\right)
$$

with

$$
\Gamma(x, s)=\left(\begin{array}{cc}
\gamma_{11}(x, s) & \gamma_{12}(x, s) \\
\gamma_{21}(x, s) & \gamma_{22}(x, s)
\end{array}\right)
$$

\subsection{Coupling with a downstream local feedback}

We now consider an open-channel with a given downstream boundary condition expressed as a local coupling between the discharge and the water elevation. This condition can either be due to a hydraulic structure, or to the effect of a semi-infinite channel.

Linearizing the relation between the discharge and the water depth leads to the following equation, expressed in the Laplace domain:

$$
q_{X}(s)=k y_{X}(s)
$$

where $k=(d Q / d Y)_{X}$ represents the local feedback.

For example, a rectangular weir is usually described by the following free flow equation:

$$
Q(X, t)=C_{d_{w}} \sqrt{2 g} L_{w}\left(Y(X, t)-Z_{w}\right)^{3 / 2}
$$

where $C_{d_{w}}$ is the discharge coefficient, $L_{w}$ the weir width, $Z_{w}$ the sill height and $g$ the gravitational acceleration. If $C_{d_{w}}$ remains constant, the feedback parameter $k$ is given here by $k=\frac{3}{2} \frac{Q}{Y(X)-Z_{w}}$.

One may note that the feedback coefficient $k$ can take any positive value, from 0 for a wall $\left(q_{X}(t)=0\right)$ to almost $\infty$ for a large reservoir $\left(y_{X}(t)=0\right)$. 
In particular, it is possible to simulate a semi-infinite channel by choosing a coefficient $k$ that approximates the non-reflective boundary condition (see [16] for details).

When assuming a uniform flow at the downstream end of the reach, the water depth at the reference flow is the normal depth $Y_{n}$, and the feedback coefficient $k_{n}$ is defined using the Manning formula.

\subsection{Saint-Venant Transfer Function}

The Saint-Venant transfer function $T F(x, s)$ at the relative distance $x$ is given by Eq. (6).

The downstream boundary adds a closed-form relation in the Laplace domain between the discharge $q_{X}(s)$ and the water depth $y_{X}(s)$ at the downstream end of the channel (Eq. (11)). This relation is coupled with Eq. (9) expressed at $x=X$, which leads to the following equation:

$$
q_{0}(s)=k_{0}(X, s) y_{0}(s)
$$

with

$$
k_{0}(X, s)=-\frac{\gamma_{12}(X, s)-k \gamma_{22}(X, s)}{\gamma_{11}(X, s)-k \gamma_{21}(X, s)}
$$

Finally Eqs. (9) and (13) lead to the Saint-Venant transfer function at any point $x$ :

$$
T F(x, s)=\gamma_{11}(x, s)+\frac{\gamma_{12}(x, s)}{k_{0}(X, s)}
$$

Eq. (15) provides a linear distributed model for flow transfer in an openchannel with a given downstream boundary condition. This model is expressed analytically in the frequency domain using a transcendental transfer function which depends on the pool characteristics. The next section presents the method used to accurately approximate this transfer function.

\section{Approximate model of flow routing with downstream local feed- back for uniform flow}

In the uniform case, [14] showed that it is possible to get a closed-form expression of the transition matrix $\Gamma(x, s)$ (see appendix A). Hence for a uniform flow, the transfer function $T F(x, s)$ is given by a closed-form expression and the moment matching method can be applied. 


\subsection{Moment matching method}

The expression of the Saint-Venant transfer function is generally too complex to be easily inverted back to the time domain explicitly, so an approximation is required to get the response in the time domain. In the following, we use the classical moment matching method (see $[9,23])$ to derive an approximate second-order-with-delay model for flow routing.

The $R$-th cumulant (i.e., logarithmic moment) of a transfer function $h$ is given by:

$$
M_{R}[h(x, t)]=(-1)^{R} \frac{d^{R}}{d s^{R}}[\log h(x, s)]_{s=0}
$$

The purpose of the moment matching method is to match the cumulants of the exact transfer function to those of the approximate one. Equating the first $n$ cumulants of the exact transfer function and the approximate one ensures a good representation for the low frequency range.

$M_{0}(x), M_{1}(x), M_{2}(x)$ and $M_{4}(x)$ denote the first four cumulants of the transfer function $T F(x, s)$ given by Eq. (15). The Taylor series expansion at $s=0$ is used for the computation of $M_{i}(x), i=0 \ldots 3$ :

$$
T F(x, s)=A(x)+B(x) s+C(x) s^{2}+D(x) s^{3}+o\left(s^{3}\right)
$$

To obtain explicit expressions of $A(x), B(x), C(x), D(x)$, a third order Taylor expansion at $s=0$ of each term of the transfer function $T F(x, s)$ is performed. Details of the computation are given in appendix B.

The relations between the cumulants and the Taylor coefficients are:

$$
\left\{\begin{array}{l}
M_{0}(x)=\log A(x) \\
M_{1}(x)=-\frac{B(x)}{A(x)} \\
M_{2}(x)=2\left(\frac{C(x)}{A(x)}-\frac{B^{2}(x)}{2 A^{2}(x)}\right) \\
M_{3}(x)=-6\left(\frac{D(x)}{A(x)}-\frac{B(x) C(x)}{A^{2}(x)}+\frac{B^{3}(x)}{3 A^{3}(x)}\right)
\end{array}\right.
$$

It is widely accepted that the flow routing in a channel is a delayed process, and that there is some attenuation of the peak flow. Such phenomenon can be accurately described by a rational transfer function with delay. To enable analytical computations, we restrict ourselves to a second-order-with-delay 
model, as it is commonly performed in the literature $([20,23])$. We show in the following that by using the moment matching method, one may identify the parameters of a second-order-with-delay that matches the low order moments of the full Saint-Venant transfer function. In some cases, as for instance for short rivers where no stable second-order is available, a first-order-with-delay model is identified.

\subsection{Second-order-with-delay}

The transfer function $T F(x, s)$ is approximated by a second-order-with-delay:

$$
T F(x, s) \approx \frac{g(x) e^{-\tau(x) s}}{\left(1+K_{1}(x) s\right)\left(1+K_{2}(x) s\right)}
$$

where $g(x), K_{1}(x), K_{2}(x)$ and $\tau(x)$ are the model parameters.

Equating the first four cumulants of the transfer function $T F(x)$ and its approximation (19) leads to:

$$
\left\{\begin{array}{l}
M_{0}(x)=\log g(x) \\
M_{1}(x)=\tau(x)+K_{1}(x)+K_{2}(x) \\
M_{2}(x)=K_{1}^{2}(x)+K_{2}^{2}(x) \\
M_{3}(x)=2 K_{1}^{3}(x)+2 K_{2}^{3}(x)
\end{array}\right.
$$

The resulting model is a second-order-with-delay (the resolution of the system provided by Eq. (20) is given in appendix D.1). It is stable only if $K_{1}(x)$ and $K_{2}(x)$ are positive, which is equivalent to $C_{S}>1$, where $C_{S}=\frac{8 C^{3}}{9 D^{2}}$. This expression of $C_{S}$ is in agreement with the one defined in [20] and corresponding to the Saint-Venant equations without the inertia terms (diffusive wave equation).

In the case where $C_{S} \leq 1$, the transfer function cannot be approximated by a stable second-order-with-delay, so the second order is replaced by a first order that is stable albeit less accurate.

\subsection{First-order-with-delay}

To approximate the transfer function by a first-order-with-delay,

$$
T F(x, s) \approx \frac{g(x) e^{-\tau(x) s}}{1+K_{1}(x) s}
$$


the time constant $K_{2}(x)$ becomes null and one has to solve the system which considers only the first three cumulants.

The solution is then given by (see appendix D.2):

$$
\left\{\begin{aligned}
\tau(x) & =M_{1}(x)-\sqrt{M_{2}(x)} \\
K_{1}(x) & =\sqrt{M_{2}(x)} \\
g(x) & =1
\end{aligned}\right.
$$

where $M_{1}(x)$ and $M_{2}(x)$ can be obtained as closed-form expression using the third order Taylor series of the transfer function given by Eq. (15).

One may note that other approximate models can be calibrated with the present method, since it simply requires the solving of the system obtained by equating the first cumulants of the transfer function and its approximation. In particular, adding a zero in the transfer function may lead to a better approximation for short canals (see [15]).

In any case, this method leads to an analytical and distributed expression of the model parameters $\left(\tau, K_{1}\right.$ for a first-order-with-delay or $\tau, K_{1}, K_{2}$ for a second-order-with-delay). These expressions provide a low frequency approximation of the flow transfer. Parameters are obtained analytically as functions of the feedback coefficient $k$ and the physical parameters of the pool (geometry, friction, discharge).

\subsection{Step response in the time domain}

Since the approximate model is a first- or a second-order-with-delay, it becomes easier to obtain the response in the time domain. Especially if the input is a step $\left(q_{0}(t)=\mathcal{H}(t)\right.$, where $\mathcal{H}$ is the Heaviside function), it is possible to obtain an analytical expression of the output.

Indeed the ordinary differential equation (ODE) corresponding to the secondorder-with-delay transfer function (Eq. (19)) is:

$$
K_{1}(x) K_{2}(x) \frac{d^{2} q}{d t^{2}}(x, t)+\left(K_{1}(x)+K_{2}(x)\right) \frac{d q}{d t}(x, t)+q(x, t)=g(x) q_{0}(t-\tau(x))
$$

For a step input and for the following initial conditions:

$$
\begin{aligned}
q(x, 0) & =0 \\
\frac{d q}{d t}(x, 0) & =0
\end{aligned}
$$


the solution is given analytically by:

$$
\begin{aligned}
q(x, t)=g(x) & {\left[\frac{K_{1}(x)}{K_{1}(x)-K_{2}(x)}\left(1-e^{-\frac{t-\tau(x)}{K_{1}(x)}}\right)\right.} \\
& \left.-\frac{K_{2}(x)}{K_{1}(x)-K_{2}(x)}\left(1-e^{-\frac{t-\tau(x)}{K_{2}(x)}}\right)\right] \mathcal{H}(t-\tau(x))
\end{aligned}
$$

If the transfer function is a first-order-with-delay (Eq. (21)), the equivalent ODE is (23) with $K_{2}(x)=0$. The initial condition is given by Eq. (24) and the solution is:

$$
q(x, t)=g(x)\left(1-e^{-\frac{t-\tau(x)}{K_{1}(x)}}\right) \mathcal{H}(t-\tau(x))
$$

In real cases, the input $q_{0}(t)$ differs from the Heaviside function. But, since the transfer function order remains low, some numerical algorithms can provide quick and accurate solvers for the ODE (23).

\section{Approximate model of flow routing with downstream local feed- back and backwater effects}

\subsection{The backwater approximation}

We now consider the discharge and the water depth variations around a nonuniform steady flow. The equilibrium regime is described by $Q(x)=Q$ and $Y(x)$, solution of the following ordinary differential equation for a boundary condition defined by downstream elevation $Y(X)$ :

$$
\frac{d Y}{d x}=\frac{S_{b}-S_{f}}{1-F^{2}}
$$

where $S_{f}$ and $F$ can be expressed as functions of $Y$.

Based on an idea initially proposed in [24], and modified in [15], we approximate a channel with a backwater curve by the concatenation of two pools. This consists in approximating the backwater curve by a stepwise linear function: a line parallel to the bed in the upstream part (corresponding to the uniform part) and a line tangent to the free surface at the downstream end in the downstream part. Let $x_{1}$ denote the abscissa of the intersection of the two lines (the discharge and the water depth variations at this point are denoted $q_{1}$ 
and $y_{1}$ respectively). The corresponding approximation of the backwater profile is schematized in Fig. 2. $S_{X}$ represents the slope of the backwater curve at the downstream end of the reach and is computed using Eq. (28).

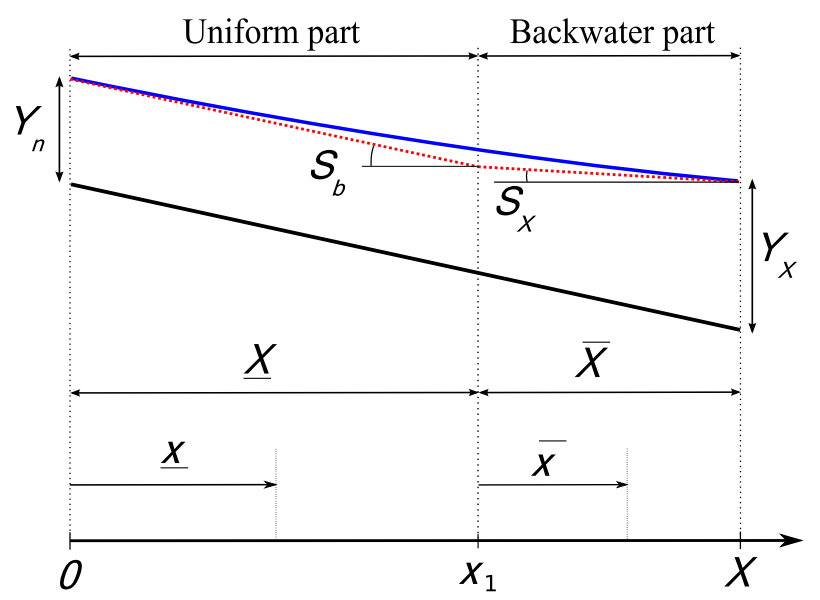

Fig. 2. Backwater curve approximation scheme.

\subsection{Transfer function for a non uniform flow}

After having divided the pool into two parts, the transfer matrix (9) can be established for each sub-pool. $\underline{\Gamma}(\underline{x}, s)$ corresponds to the uniform part (length $\underline{X}=x_{1}$, relative position $\left.\underline{x}=x\right)$, and $\bar{\Gamma}(\bar{x}, s)$ corresponds to the backwater part (length $\bar{X}=X-x_{1}$, relative position $\bar{x}=x-x_{1}$ ).

In the uniform part $\left(0 \leq x \leq x_{1}\right)$, we have:

$$
\left(\begin{array}{l}
q(x, s) \\
y(x, s)
\end{array}\right)=\underline{\Gamma}(\underline{x}, s)\left(\begin{array}{l}
q_{0}(s) \\
y_{0}(s)
\end{array}\right)
$$

and in the backwater part $\left(x_{1} \leq x \leq X\right)$ :

$$
\left(\begin{array}{l}
q(x, s) \\
y(x, s)
\end{array}\right)=\bar{\Gamma}(\bar{x}, s)\left(\begin{array}{l}
q_{1}(s) \\
y_{1}(s)
\end{array}\right)
$$

So it is possible to define an equivalent transfer matrix $\hat{\Gamma}(x, s)$ at any relative distance $0 \leq x \leq X$ :

$$
\left(\begin{array}{l}
q(x, s) \\
y(x, s)
\end{array}\right)=\hat{\Gamma}(x, s)\left(\begin{array}{l}
q_{0}(s) \\
y_{0}(s)
\end{array}\right)
$$


with

$$
\hat{\Gamma}(x, s)= \begin{cases}\underline{\Gamma}(\underline{x}, s) & \text { if } 0 \leq x \leq x_{1} \\ \bar{\Gamma}(\bar{x}, s) \underline{\Gamma}(\underline{X}, s) & \text { if } x_{1} \leq x \leq X\end{cases}
$$

As in section 2.7, coupling the feedback relation (Eq. (11)) and Eq. (31) leads to:

$$
T F(x, s)=\hat{\gamma}_{11}(x, s)+\frac{\hat{\gamma}_{12}(x, s)}{\hat{k}_{0}(X, s)}
$$

with

$$
\hat{k}_{0}(X, s)=-\frac{\hat{\gamma}_{12}(X, s)-k \hat{\gamma}_{22}(X, s)}{\hat{\gamma}_{11}(X, s)-k \hat{\gamma}_{21}(X, s)}
$$

Eq. (33) gives to a closed-form expression of the transfer function $T F(x, s)$ at any relative distance $x(0 \leq x \leq X)$, depending on the pool characteristics. It provides a linear distributed model for flow transfer in an open-channel with a given downstream boundary condition and a non-uniform flow.

\subsection{Approximate model}

The method used to approximate the transfer function for non-uniform flow is the same method as in section 3. As we know the exact linear transfer function obtained in the previous section, we compute the first cumulants (see appendix C) and apply the moment matching method.

\section{Validation}

\subsection{Simulations}

For validation purposes, we consider a trapezoidal prismatic channel, with characteristics detailed in table 1 , where $X$ is the channel length $(\mathrm{m}), m$ the bank slope $(\mathrm{m} / \mathrm{m}), B$ the bed width $(\mathrm{m}), S_{b}$ the bed slope $(\mathrm{m} / \mathrm{m}), n$ the Manning roughness coefficient $\left(\mathrm{sm}^{-1 / 3}\right), Q$ the reference discharge $\left(\mathrm{m}^{3} \mathrm{~s}^{-1}\right), Y_{n}$ the normal depth $(\mathrm{m})$ and $k_{n}$ the feedback coefficient $\left(\mathrm{m}^{2} \mathrm{~s}^{-1}\right)$ corresponding to the reference discharge $Q$.

Table 1

Parameters of the example canal

\begin{tabular}{|c|c|c|c|c|c|c|c|}
\hline$X$ & $m$ & $B$ & $S_{b}$ & $n$ & $Q$ & $Y_{n}$ & $k_{n}$ \\
\hline \hline 10000 & 1 & 50 & 0.0002 & 0.02 & 100 & 1.87 & 88.8 \\
\hline
\end{tabular}


In order to validate our model and analyze the effects of the downstream boundary condition, different situations have been simulated with three different models. For each situation the Bode diagram and the response to a step are plotted for two relative positions: in the middle of the channel $(X / 2)$ and at the downstream end $(X)$.

The Bode diagram is a representation of the transfer function in the frequency domain, used to analyzed the behavior of the system in low and high frequencies. It represents the magnitude $A_{d B}$ (in decibel) and the phase $\phi$ (in degree) of the transfer function at $s=i \omega$ (where $i^{2}=-1$ ).

$$
\left\{\begin{aligned}
A_{d B}(x, \omega) & =20 \log |T F(x, i \omega)| \\
\phi(x, \omega) & =\arg (T F(x, i \omega))
\end{aligned}\right.
$$

The step response is the response of the transfer function to a step input defined as follows:

$$
q_{0}(t)= \begin{cases}0 & \text { if } t<0 \\ 1 & \text { if } t \geq 0\end{cases}
$$

The first model is the linearized Saint-Venant model which is used as a reference for linear flow transfer. Its Bode diagram is obtained using the transfer function established in section 4.2, and the response in the time domain is simulated by SIC ([2]), a software that discretizes the Saint-Venant equations using a Preissmann scheme. The second model is the LBLR, our second-orderwith-delay model (or first-order-with-delay if there is no stable second order solution), which considers a finite channel with a downstream boundary condition. The third one is a model assuming a semi-infinite channel by neglecting the upward waves, so that the flow is uniform and downstream structures have no effect upstream. This model, developed in [9], is based on the transfer function $T F(x)=e^{\lambda_{1} x}$ (see appendix A for the definition of $\lambda_{1}$ ), approximated by a second-order-with-delay using the moment matching method described in section 3.1 .

The validation takes place in three steps corresponding to three different downstream boundary conditions. Firstly, we consider a large reservoir or a large lake, in which the downstream water depth remains constant, which means that $k \rightarrow \infty$ (Eq. 11). The value $Y_{X}=1.5 Y_{n}=2.81 \mathrm{~m}$ has been chosen for this simulation.

Secondly, a gate is introduced at the downstream end of the reach in order to analyze the effects of a cross structure. This gate is described by Eq. (37). The characteristics of the gate are listed in table 2 , where $L_{g}$ is the width of the gate $(\mathrm{m}), W_{g}$ is the gate opening $(\mathrm{m})$ and $C_{d_{g}}$ is the discharge coefficient. 
The downstream boundary condition becomes: $Y_{X}=1.12 Y_{n}$ and $k=0.27 k_{n}$.

$$
Q(X, t)=C_{d_{g}} L_{g} W_{g} \sqrt{2 g Y(X, t)}
$$

Table 2

Characteristics of the downstream gate

\begin{tabular}{|c|c|c|}
\hline$L_{g}$ & $W_{g}$ & $C_{d_{g}}$ \\
\hline \hline 40 & 0.65 & 0.6 \\
\hline
\end{tabular}

Lastly, the gate is replaced by a weir described by Eq. (12). Its characteristics are listed in table 3 , where $L_{w}$ is the width of the weir $(\mathrm{m}), Z_{w}$ is the sill height $(\mathrm{m})$ and $C_{d_{w}}$ is the discharge coefficient. For this case, the downstream boundary condition is: $Y_{X}=1.74 Y_{n}$ and $k=1.34 k_{n}$.

Table 3

Characteristics of the downstream weir

\begin{tabular}{|c|c|c|}
\hline$L_{w}$ & $Z_{w}$ & $C_{d_{w}}$ \\
\hline \hline 40 & 2 & 0.4 \\
\hline
\end{tabular}

\subsection{Reservoir at the downstream end}

The first simulation considers a large reservoir as the downstream boundary condition, defined by $Y_{X}=1.5 Y_{n}$ and $k \rightarrow \infty$. Fig. 3 represents the backwater curve and its approximation for this case, showing the non-uniformity of the flow. Figs. 4 and 5 show the Bode diagram and the step response of the three models.

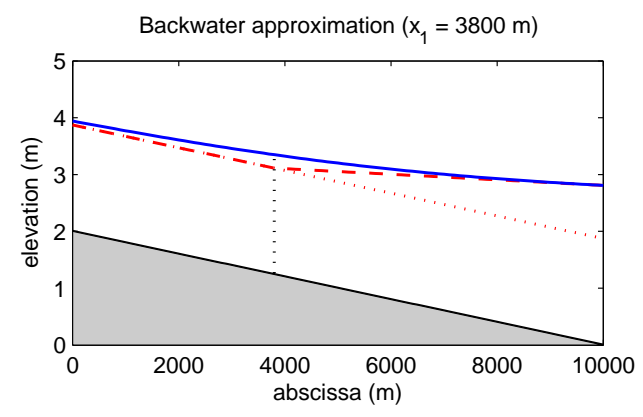

Fig. 3. Backwater curve and its approximation with a reservoir at the downstream end. Real backwater curve (-), uniform normal depth $Y_{n}(\cdots)$ and approximate backwater curve $(--) \cdot x_{1}$ is also represented.

The semi-infinite model represents a uniform flow without feedback, and is insensitive to the downstream boundary condition. The Bode diagrams in Fig. 4 show that the complete Saint-Venant model significantly differs from the semiinfinite model when considering a non-uniform flow with infinite feedback. The 

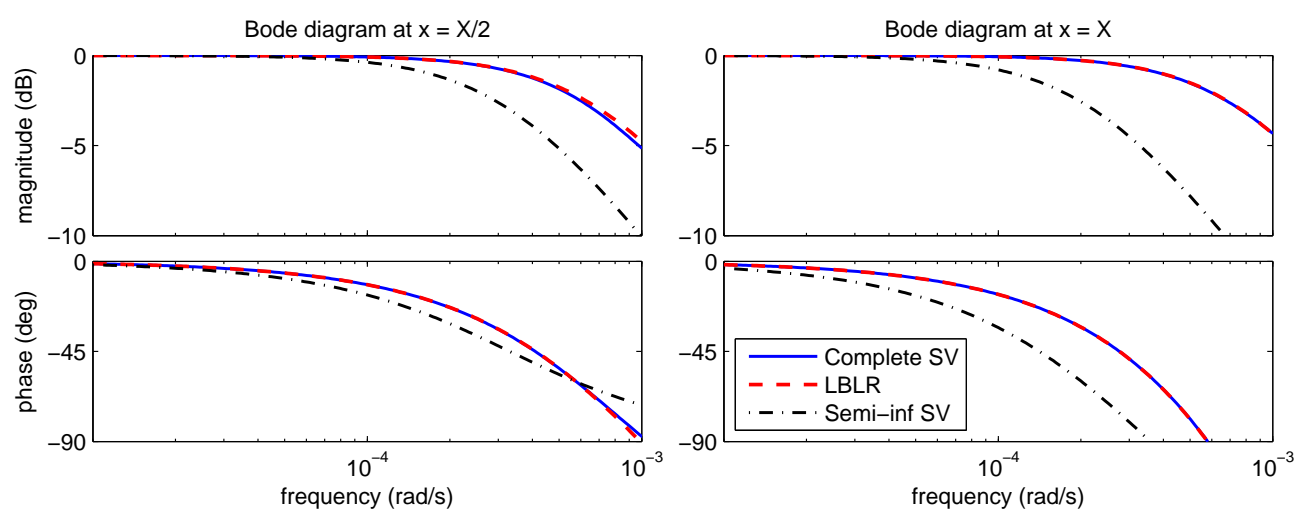

Fig. 4. Bode diagram at $X / 2$ and $X$ with a reservoir at the downstream end.
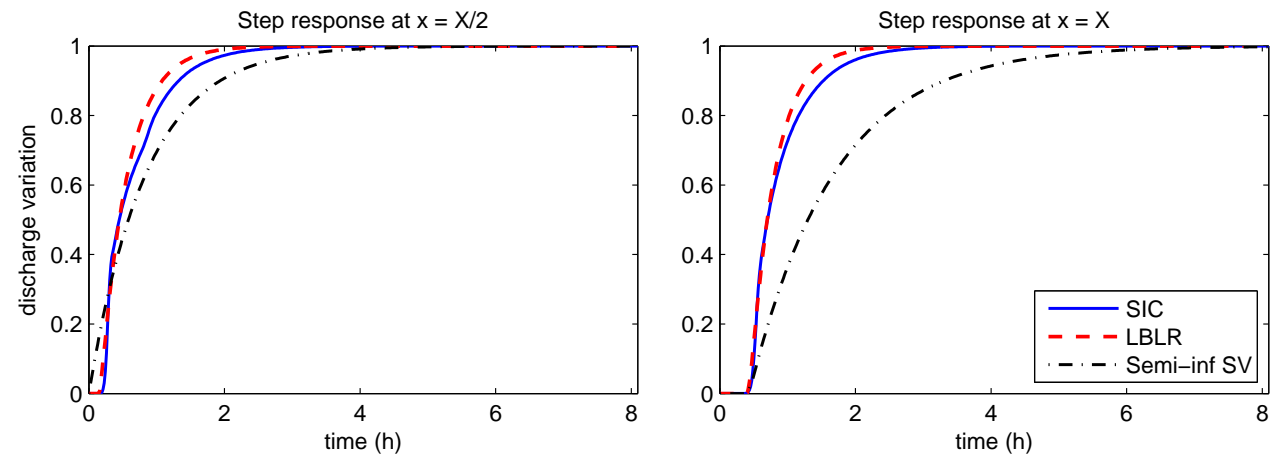

Fig. 5. Step response at $X / 2$ and $X$ with a reservoir at the downstream end.

LBLR model satisfactorily reproduces these changes, at both location $X / 2$ and $X$. The differences are also visible in the time domain in Fig. 5, where LBLR response remains close to that of SIC.

\subsection{Cross structure effect: a gate at the downstream end}

In the second simulation, a gate is added at the downstream end of the channel reach. Characteristics of the gate are listed in table 2. Fig. 6 represents the backwater curve and its approximation for this case. Figs. 7 and 8 show the Bode diagram and the step response of the two approximate models and the complete Saint-Venant model.

In this case, the downstream water depth is close to normal depth. Hence, the non-uniform part is very short (see Fig. 6), and backwater effects are negligible. Consequently, the difference shown at the downstream end (in the frequency domain and in the time domain) is essentially due to feedback effects. The LBLR model satisfactorily takes these effects into account. One can also note that the transfer at $x=X / 2$ is not significantly affected by the change in the downstream boundary condition (see left-hand side of Fig. 7), whereas 


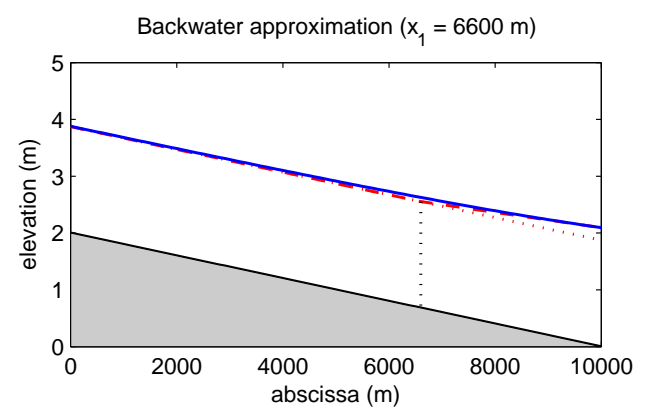

Fig. 6. Backwater curve and its approximation with a gate at the downstream end. Real backwater curve (-), uniform normal depth $Y_{n}(\cdots)$ and approximate backwater curve $(--) \cdot x_{1}$ is also represented.
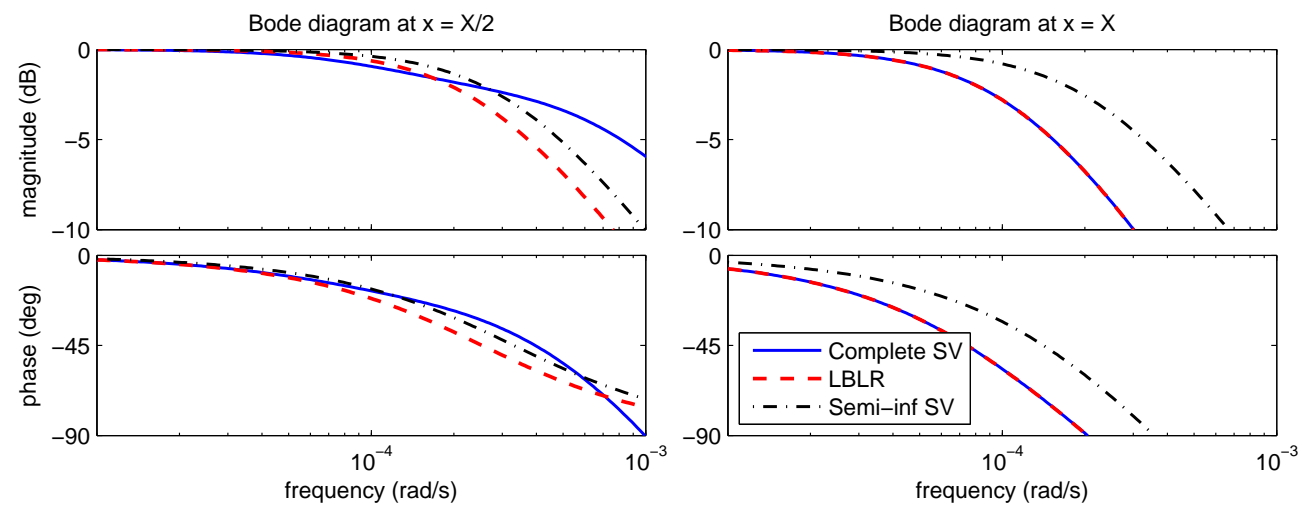

Fig. 7. Bode diagram at $X / 2$ and $X$ with a gate at the downstream end.
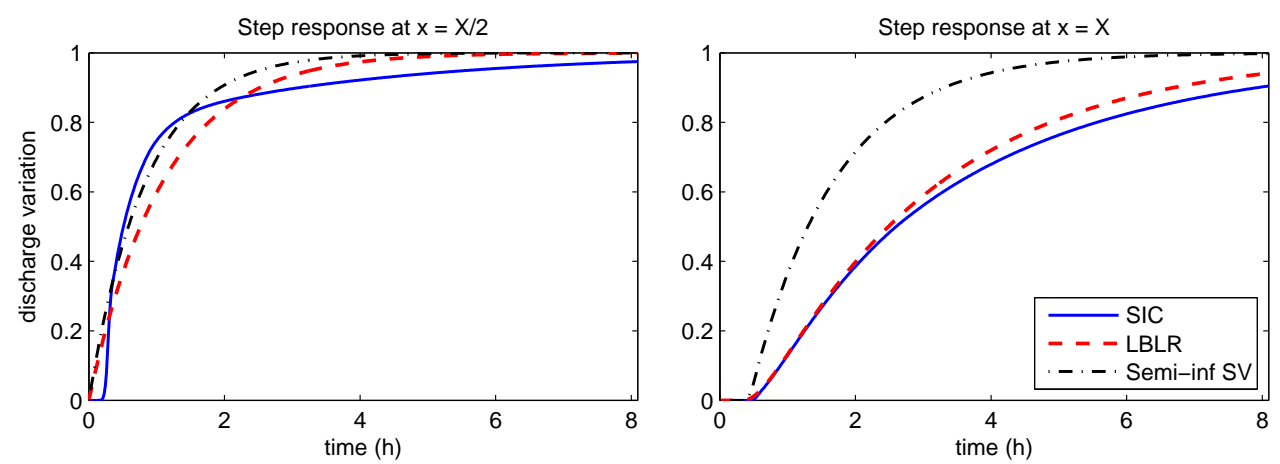

Fig. 8. Step response at $X / 2$ and $X$ with a gate at the downstream end.

it has a greater impact on transfer at the downstream end of the reach (see right-hand side of Fig. 7). Lastly, the magnitude of the complete Saint-Venant solution in the high frequency is higher at $X / 2$ than at $X$. Yet, the semiinfinite model and the LBLR model are first or second orders based on a low frequency approximation. Their responses logically differ a little from the complete Saint-Venant one at $x=X / 2$, where higher frequencies are solicited. 


\subsection{Cross structure effect: a weir at the downstream end}

In the last simulation, the gate is replaced by a weir. Its characteristics are listed in table 3. Fig. 9 represents the backwater curve and its approximation for this case. Figs. 10 and 11 show the Bode diagram and the step response of the two approximate models and the complete Saint-Venant model.

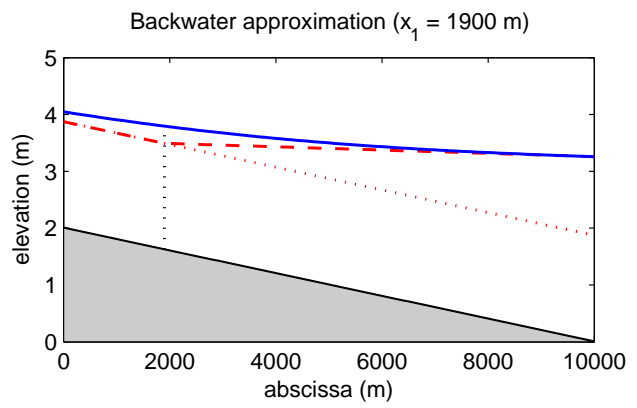

Fig. 9. Backwater curve and its approximation with a weir at the downstream end. Real backwater curve (-), uniform normal depth $Y_{n}(\cdots)$ and approximate backwater curve $(--)$. Abscissa $x_{1}$ is also represented.
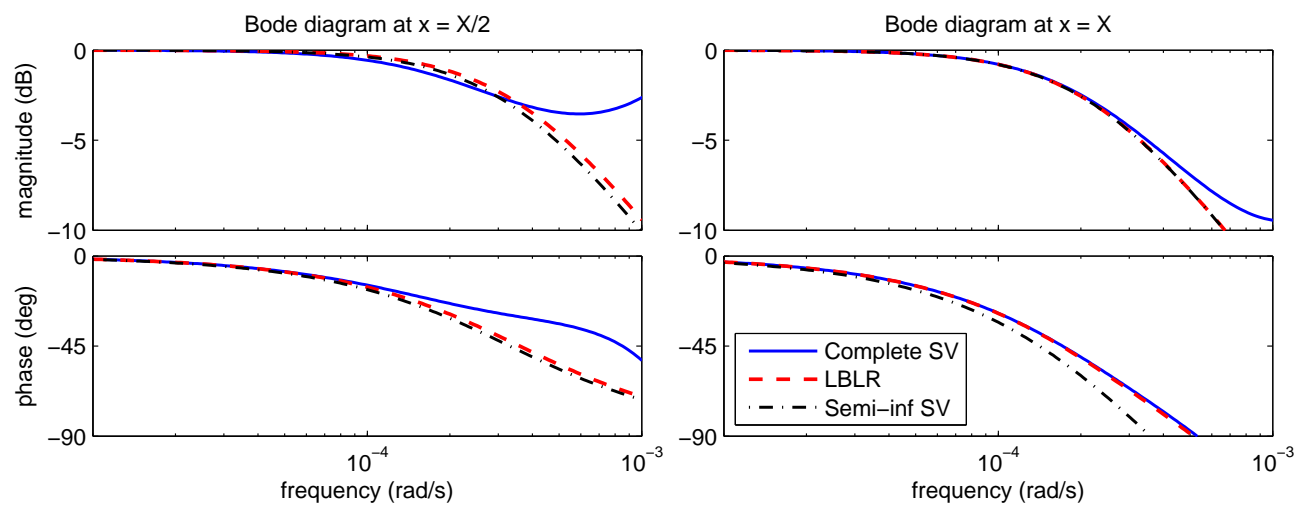

Fig. 10. Bode diagram at $X / 2$ and $X$ with a weir at the downstream end.
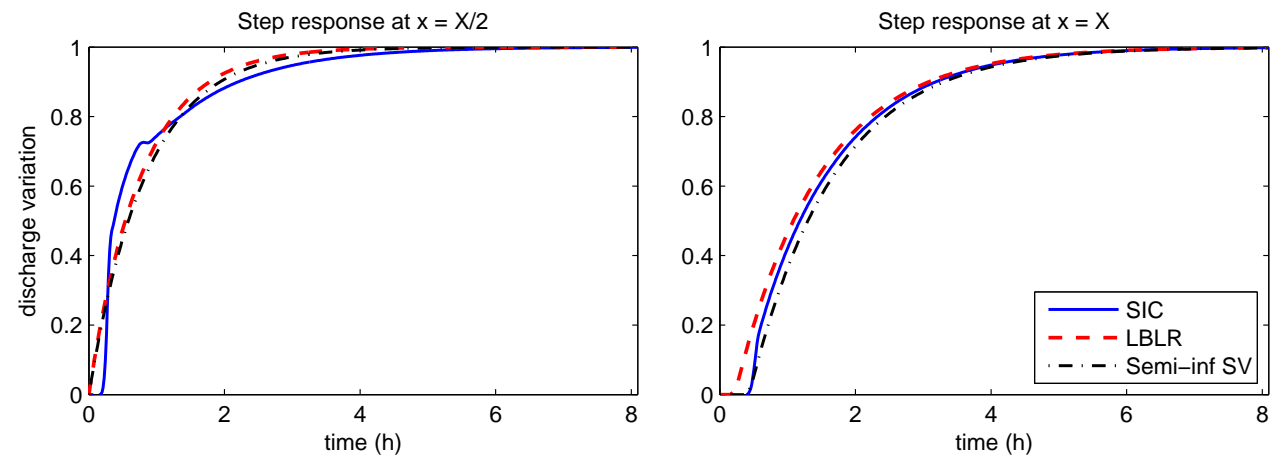

Fig. 11. Step response at $X / 2$ and $X$ with a weir at the downstream end.

Fig. 9 shows that this downstream boundary condition imposes a largely nonuniform flow, so that backwater effects are non negligible. Yet, the semi-infinite 
model and the LBLR model are very close to the complete Saint-Venant model in the low frequency range, and in the time domain, as much in the middle of the reach as at the downstream end. This simulation shows that backwater effects can be compensated by feedback effects. In fact, the feedback coefficient $k$ in this case is greater than the one in the uniform case $k_{n}\left(k=1.34 k_{n}\right)$, which accelerates the flow dynamics (see the case of a reservoir in section 5.2). On the other hand, backwater effects are responsible for a deceleration of the dynamics, which compensates for the feedback effects. In some cases, like this one, the semi-infinite model could be sufficient to reproduce the dynamics despite the presence of a hydraulic structure at the downstream end.

\section{Summary and discussion}

\subsection{Discussion: response time to a step inflow}

The downstream boundary condition, usually neglected in flow routing methods which merely consider a semi-infinite channel and a uniform flow, may significantly influence flow dynamics. The moment matching method on the linearized Saint-Venant transfer function coupled with the linearized feedback equation at the downstream boundary allowed us to build a new approximate model, a second-order-with-delay called LBLR. The delay time $\tau$ and the time constants $K_{1}$ and $K_{2}$ are expressed analytically as closed-form expressions of the pool characteristics (geometry, friction, discharge, downstream water depth, and the feedback parameter).

Results show that the LBLR model satisfactorily takes into account the effects of the downstream boundary condition. Indeed the LBLR solution accurately matches the one of the complete Saint-Venant model when the downstream conditions vary (feedback or backwater effects), while the semi-infinite SaintVenant model does not react to those variations. This difference can be quantified by measuring the response time at $80 \%$, which corresponds to the time when the downstream discharge variation reaches $0.8 \mathrm{~m}^{3} / \mathrm{s}$ out of a step input of $1 \mathrm{~m}^{3} / \mathrm{s}$. Table 4 summarizes the response time $\left(\mathrm{RT}_{80 \%}\right)$ at $x=X$ for each model and each simulation, and the relative error $\left(\epsilon_{r}\right)$ with respect to the complete Saint-Venant model.

Finally, the LBLR model greatly improves the estimation of the flow dynamics in canals possessing a simple geometry, by providing closed-form expressions of the coefficients that describe the chosen approximate model (e.g. a secondorder-with-delay). The model is obtained via three main approximations which are the limitations of the method: the low frequency approximation (moment matching method), the backwater curve approximation and the linearization 
Table 4

Response time at $80 \%\left(\mathrm{RT}_{80 \%}\right)$ at $x=X$ for each model and each simulation and relative error $\left(\epsilon_{r}\right)$ with respect to the complete Saint-Venant model.

\begin{tabular}{|c||c|c|c|c|c|c|}
\hline \multicolumn{1}{|c||}{ Model } & \multicolumn{2}{c|}{ SIC } & \multicolumn{2}{c|}{ LBLR } & \multicolumn{2}{c|}{ Semi-infinite } \\
\hline Simulation & $\mathrm{RT}_{80 \%}$ & $\epsilon_{r}$ & $\mathrm{RT}_{80 \%}$ & $\epsilon_{r}$ & $\mathrm{RT}_{80 \%}$ & $\epsilon_{r}$ \\
\hline \hline Reservoir & $1.17 \mathrm{~h}$ & - & $1.04 \mathrm{~h}$ & $11 \%$ & $2.44 \mathrm{~h}$ & $109 \%$ \\
\hline Gate & $5.57 \mathrm{~h}$ & - & $4.89 \mathrm{~h}$ & $12 \%$ & $2.44 \mathrm{~h}$ & $56 \%$ \\
\hline Weir & $2.33 \mathrm{~h}$ & - & $2.23 \mathrm{~h}$ & $4 \%$ & $2.44 \mathrm{~h}$ & $5 \%$ \\
\hline
\end{tabular}

around a steady state flow. These approximations may explain the minor difference observed on the graphs between the responses of the LBLR model and the complete Saint-Venant one.

\subsection{Variations of model parameters as functions of the discharge}

The time constants $\tau, K_{1}, K_{2}$ of the approximate transfer function can be expressed as closed-form expressions of the reference discharge $Q$. To represent this, an abacus can be drawn, which represents these constants with respect to the reference discharge for a given downstream boundary condition and at a given position in the channel. For instance, Fig. 12 shows the variations of the time constants with respect to the reference discharge at the downstream end of the reach in the uniform case.

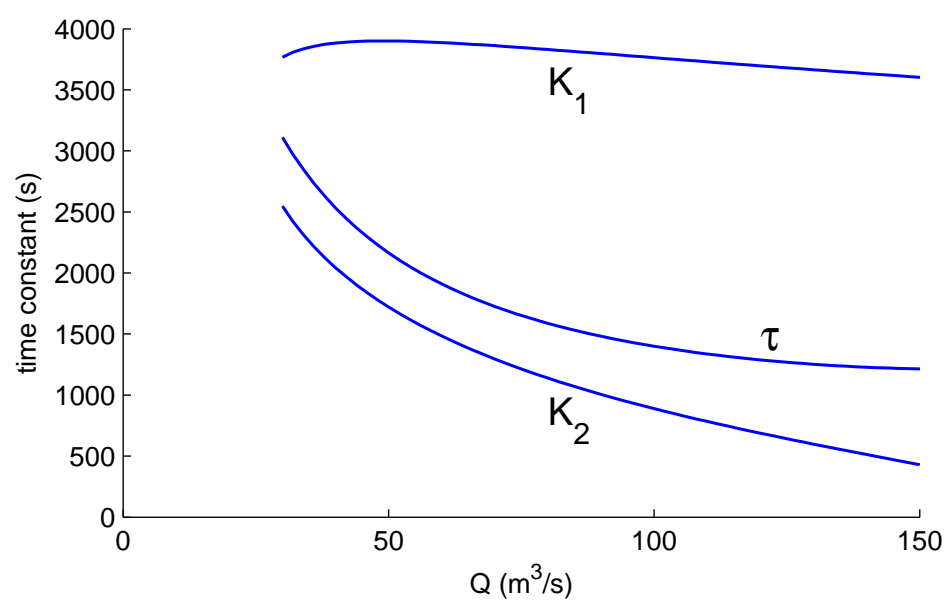

Fig. 12. Evolution of the coefficients $\tau, K_{1}, K_{2}$ with respect to the reference discharge $Q$, at the downstream end of the reach.

As we expected, the time-delay $\tau$ decreases when the discharge $Q$ increases. Similarly, the response time can be given as a closed-form expression, and an abacus can be drawn representing the response time with respect to the reference discharge and a given downstream boundary condition. 
Through the time constants variations, this graph shows the influence of the chosen reference discharge on the flow dynamics. This is a consequence of the non-linearity of the Saint-Venant equations. A non-linear extension is envisaged, following either a multilinear approach (e.g. $[3,5,12,22]$ ) or a non-linear extension (e.g. [18]). However, we will see in the next section that the linear assumption may be sufficient to capture the main features of the flow routing process.

\subsection{Attenuation in flood propagation}

Two important characteristics of flood propagation are the attenuation level of the peak flow and the peak time. In this section, the previous example canal is considered with a weir at the downstream end. The weir is $80 \mathrm{~m}$ long, and $2 \mathrm{~m}$ high, with a discharge coefficient of 0.4 . Eq. (12) is used to characterize the weir. The upstream discharge routed through the channel is defined as follows:

$$
Q(0, t)=Q_{m}+\left(Q_{M}-Q_{m}\right) \frac{t}{T_{0}} e^{1-\frac{t}{T_{0}}} \quad \text { if } t \geq 0
$$

where $Q_{m}=20 \mathrm{~m}^{3} / \mathrm{s}$ and $Q_{M}=120 \mathrm{~m}^{3} / \mathrm{s}$ are the minimum and maximum discharges respectively, and $T_{0}=2 \mathrm{~h}$ is the upstream time to peak. The reference discharge is set to the mean value of the upstream discharge: $Q=56$ $\mathrm{m}^{3} / \mathrm{s}$. With the chosen downstream boundary condition, this leads to $Y_{X}=$ $1.92 Y_{n}$ and $k=2.21 k_{n}$. Fig. 13 shows the response to the upstream hydrograph at $x=X / 2$ and $x=X$.
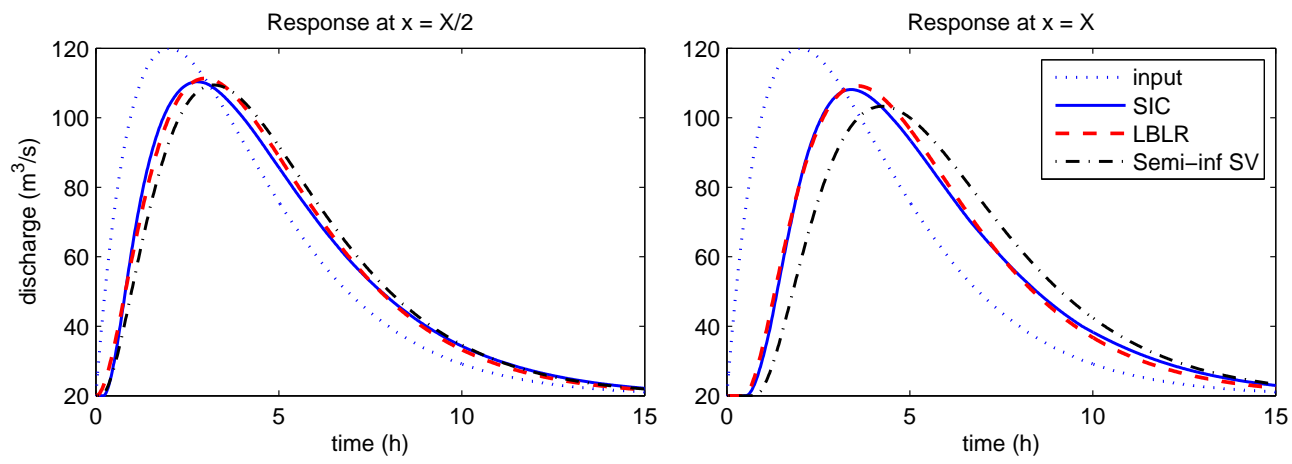

Fig. 13. Simulation of the response in the time domain at $x=X / 2$ and $x=X$.

The attenuation level is defined as the difference between the upstream maximum discharge and the maximum discharge at the abscissa $x$. Table 5 summarizes, for each simulation, the attenuation level and the peak time at the downstream end of the channel $(x=X)$. The relative error with respect to SIC results is given in brackets. 
Table 5

Attenuation level and peak time at $x=X$ for each simulation. Relative error with respect to SIC results is given in brackets.

\begin{tabular}{|c||c|c|c|}
\hline Model & SIC & LBLR & Semi-infinite \\
\hline \hline attenuation $\left(\mathrm{m}^{3} / \mathrm{s}\right)$ & 11.9 & $10.8(9.2 \%)$ & $16.7(40.3 \%)$ \\
\hline peak time $(\mathrm{h})$ & 3.42 & $3.57(4.4 \%)$ & $4.26(24.6 \%)$ \\
\hline
\end{tabular}

For this realistic example case, accounting for the downstream boundary condition leads to a great improvement in the simulation of peak flow attenuation. In addition, although the upstream discharge varies from 20 to $120 \mathrm{~m}^{3} / \mathrm{s}$ in this simulation, the linearization of the Saint-Venant equations seems to be still valid according to the good results obtained with the LBLR model. Let us also notice that the peak time is correctly reproduced by the LBLR model, while it is largely overestimated by the model which uses the semi infinite assumption.

\subsection{Criteria on the downstream boundary condition}

It is usual in the literature to perform simulation on longer river stretches in order to minimize the effects of the downstream boundary condition (see e.g. [4]). In that case, the semi-infinite canal is usually simulated with a three to five times longer canal. The LBLR model can be used to provide a quantification of this approximation. For instance, let us consider the response time (defined in section 6.1) for the example canal. We first estimate the response time at the distance $x=\bar{X}=10000 \mathrm{~m}$ calculated with the semi-infinite Saint-Venant model and denoted $\mathrm{RT}_{\text {semi-inf }}$. Then we vary the downstream water depth $Y_{X}$ and the feedback coefficient $k$ in the intervals $\left[0.5 Y_{n}, 4 Y_{n}\right]$ and $\left[0.1 k_{n}, 3 k_{n}\right]$, respectively. Each couple $\left(Y_{X}, k\right)$ represents a particular downstream boundary condition. We expect that the response time at $x=\bar{X}$, denoted $\mathrm{RT}_{L B L R}$, calculated with the LBLR model tends to $\mathrm{RT}_{\text {semi-inf }}$ when the length of the canal $X$ increases. We define the distance $X_{\infty}$ as the minimum length $X$ from which the relative error between $\mathrm{RT}_{\text {semi-inf }}$ and $\mathrm{RT}_{L B L R}$ is lower than $5 \%$.

Fig. 14 shows the evolution of $X_{\infty} / \bar{X}$ with respect to $Y_{X} / Y_{n}$ and $k / k_{n}$. $X_{\infty} / \bar{X}=1$ means that the downstream boundary condition has almost no effect on the dynamics. On the contrary, $X_{\infty} / \bar{X}=5$ means that the length of the canal has to be multiplied by 5 to ensure an error on the response time lower than $5 \%$.

This graph shows that backwater effects as well as feedback effects can have a large impact on the dynamics. The black zone corresponds to the cases where those two effects are compensated, which means that the channel has quite the same behavior with or without the downstream boundary condition. One 


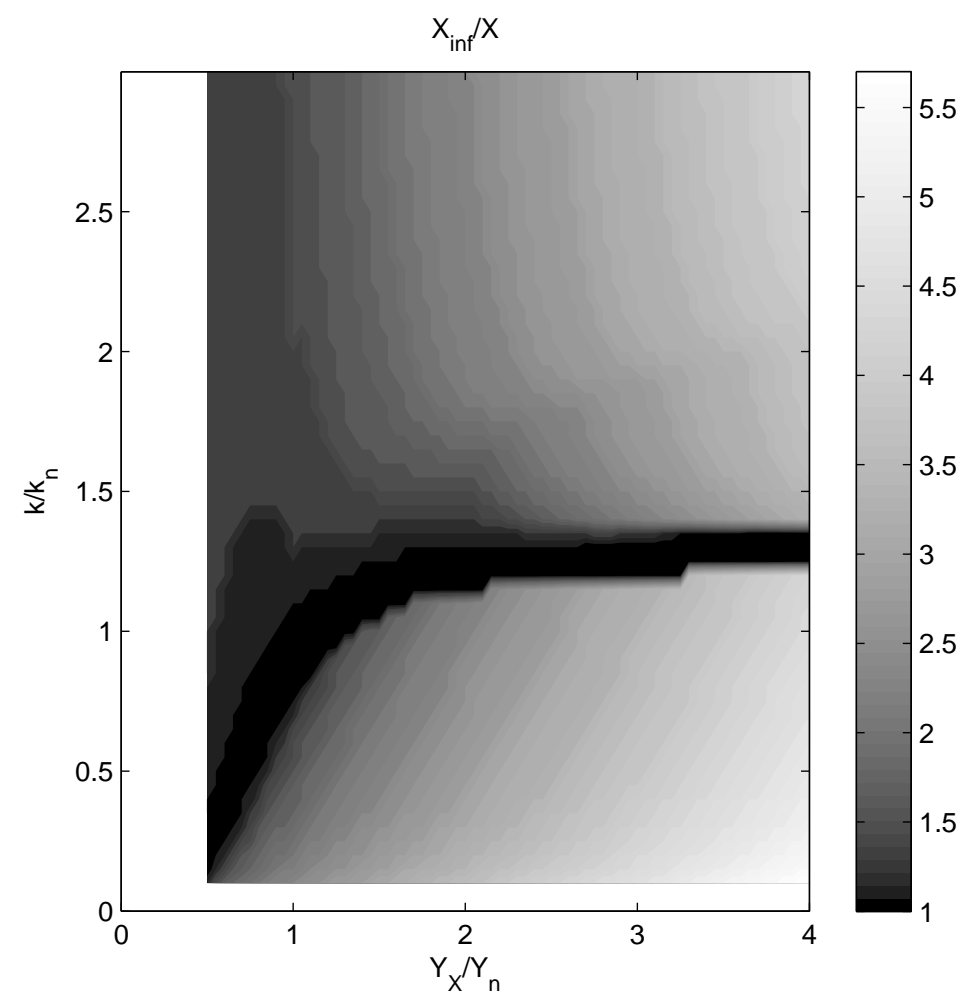

Fig. 14. Evolution of $X_{\infty} / \bar{X}$ with respect to $Y_{X} / Y_{n}$ and $k / k_{n}$.

may conclude that, even under normal flow conditions $\left(Y_{X}=Y_{n}\right)$, feedback effect can have an important impact on dynamics, especially for low values of the feedback coefficient $k$. The graph also leads to the other conclusion that for the particular value of $k=1.3 k_{n}$, backwater effects have almost no impact on the dynamics, as shown previously with the canal ended by a weir. For the flood routing process, similar criteria can be built based on the calculation of the peak time or of the attenuation of the peak discharge.

\section{Conclusion}

The article proposes a new analytical and distributed model to approximate flow transfer for a non-uniform open-channel pool. The LBLR is a first- or second-order-with-delay transfer function that approximates the complete transfer matrix of the linearized Saint-Venant equations coupled with a downstream boundary condition. The model has been shown to efficiently reproduce the dynamic behavior of an open-channel with backwater and different downstream boundary conditions.

The main advantages of this model are that it gives the discharge at any location in the channel depending on the discharge at the upstream end, it 
integrates the flow non-uniformity due to downstream hydraulic structures, and results are given as closed-form expressions, so quick computations are made possible.

Many applications are possible, from an accurate flow routing to the estimation of the response time or the attenuation level in a prismatic open-channel, even in the case of large discharge variations. The research of equivalent geometric characteristics for non prismatic channels is under study. At the same time, this model remains simple enough to be used for controller design for openchannel, such as downstream PI controllers, or even for advanced controller design (e.g. multivariable controllers).

\section{Acknowledgment}

This work was jointly supported by Region Languedoc Roussillon and Cemagref within the $\mathrm{PhD}$ thesis of Simon Munier, under the supervision of X. Litrico and G. Belaud.

\section{References}

[1] Balogun OS, Hubbard M, DeVries JJ. Automatic control of canal flow using linear quadratic regulator theory. Journal of Hydraulic Engineering, 1988;114:75-102.

[2] Baume JP, Malaterre PO, Belaud G, Le Guennec B. SIC: a 1D Hydrodynamic Model for River and Irrigation Canal Modeling and Regulation. Métodos Numéricos em Recursos Hidricos, 2005;7:1-81.

[3] Becker A, Kundzewicz ZW. Nonlinear flood routing with multilinear models. Water Resources Research, 1987;23(6):1043-1048.

[4] Bentura PLF, Michel C. Flood routing in a wide channel with a quadratic lag-and-route method. Hydrological Sciences, 1997;42(2):169-189.

[5] Camacho LA, Lees MJ. Multilinear discrete lag-cascade model for channel routing. Journal of Hydrology, 1999;226(1-2):30-47.

[6] Chow V. Open-channel Hydraulics. McGraw-Hill Book Company, New York. 1988. $680 \mathrm{p}$.

[7] Chung WH, Aldama AA, Smith JA. On the effects of downstream boundary conditions on diffusive flood routing. Advances in Water Resources, 1993;16(5):259-275. 
[8] Dooge J, Napiórkowski J, Strupczewski W. The linear downstream response of a generalized uniform channel. Acta Geophysica Polonica, 1987a;XXXV(3):279293.

[9] Dooge J, Napiórkowski J, Strupczewski W. Properties of the generalized downstream channel response. Acta Geophysica Polonica, 1987b;XXXV(6):967972 .

[10] Garcia A, Hubbard M, DeVries JJ. Open channel transient flow control by discrete time LQR methods. Automatica, 1992;28:255-264.

[11] Hurand, P. and Kosuth, P. Régulations en rivière. La Houille Blanche, 1993;2/3:143-149.

[12] Kundzewicz ZW. Multilinear flood routing. Acta Geophysica Polonica, 1984;32(4):419-445.

[13] Litrico, X. Robust IMC flow control of SIMO dam-river open-channel systems. IEEE Trans. on Control Systems Technology, 2002;10:432-437.

[14] Litrico X, Fromion V. Frequency modeling of open channel flow. Journal of Hydraulic Engineering, 2004a;130(8):806-815.

[15] Litrico X, Fromion V. Simplified modeling of irrigation canals for controller design. Journal of Irrigation and Drainage Engineering, 2004b;130(5):373-383.

[16] Litrico X, Fromion V. Boundary control of linearized Saint-Venant equations oscillating modes. Automatica, 2006;42(6):967-972.

[17] Litrico, X. and Georges, D. Robust continuous-time and discrete-time flow control of a dam-river system (I): Modelling \& (II): Controller Design. Applied Mathematical Modelling, 1999;23:809-827,829-846.

[18] Litrico X, Pomet JB. Nonlinear modeling and control of a long river stretch. European Control Conference. 2003.

[19] Lundberg KH, Miller HR, Trumper DL. Initial conditions, generalized functions, and the Laplace transform: Troubles at the origin. IEEE Control Systems Magazine, 2007;27(1):22-35.

[20] Malaterre, PO. Modélisation analyse et commande optimale LQR d'un canal d'irrigation. M. Sc. thesis, LAAS - CNRS - ENGREF - Cemagref. 1994. In French.

[21] Malaterre, PO. PILOTE: Linear quadratic optimal controller for irrigation canals. J. Irrig. Drain. Eng., 1998;124:187-194.

[22] Perumal M. Multilinear discrete cascade model for channel routing. Journal of Hydrology, 1994;158(1-2):135-150.

[23] Rey J. Contribution à la modélisation et la régulation des transferts d'eau sur des systèmes de type rivière/baches intermédiaires. M. Sc. thesis, Université Montpellier 2. 1990. In French. 
[24] Schuurmans J, Clemmens AJ, Dijkstra S, Hof A, Brouwer R. Modeling of irrigation and drainage canals for controller design. Journal of Irrigation and Drainage Engineering, 1999;125(6):338-344.

[25] Strelkoff TS, Deltour JL, Burt CM, Clemmens AJ, Baume JP. Influence of Canal Geometry and Dynamics on Controllability. Journal of Irrigation and Drainage Engineering, 1998;124(1):16-22.

[26] Tsai CW. Applicability of kinematic, noninertia, and quasi-steady dynamic wave models to unsteady flow routing. Journal of Hydraulic Engineering, 2003;129(8):613-627.

[27] Tsai CW. Flood routing in mild-sloped rivers-wave characteristics and downstream backwater effect. Journal of Hydrology, 2005;308(1-4):151-167.

[28] Weinmann P, Laurenson E. Approximate flood routing methods: A review. Journal of the Hydraulics Division, ASCE, 1979;105:1521-1536. 


\section{Appendix}

\section{A Computation of Saint-Venant Transfer Matrix}

Applying Laplace transform to the linear partial differential Eqs. (2) and (3), and reordering leads to an ordinary differential equation in the variable $x$, with a complex parameter $s$ (the Laplace variable):

$$
\frac{d}{d x}\left(\begin{array}{l}
q(x, s) \\
y(x, s)
\end{array}\right)=\mathcal{A}_{s}\left(\begin{array}{l}
q(x, s) \\
y(x, s)
\end{array}\right)
$$

with $\mathcal{A}_{s}=\left(\begin{array}{cc}0 & -T s \\ -\frac{s-\mu}{T\left(\mathcal{C}^{2}-V^{2}\right)} & \frac{2 V T s+\nu}{T\left(\mathcal{C}^{2}-V^{2}\right)}\end{array}\right)$

There are two boundary conditions $q(0, s)$ in $x=0$ and $q(X, s)$ in $x=X$. Let us consider the integration of this linear system

$$
\frac{d \xi_{s}(x)}{d x}=\mathcal{A}_{s} \xi_{s}(x)
$$

with $\xi_{s}(x)=(q(x, s), y(x, s))^{T}$ and where the initial condition is defined at $x=0$. The solution of the system always exists and is given by:

$$
\xi_{s}(x)=\Gamma_{s}(x) \xi_{s 0}=\left(\begin{array}{cc}
\gamma_{11}(x, s) & \gamma_{12}(x, s) \\
\gamma_{21}(x, s) & \gamma_{22}(x, s)
\end{array}\right) \xi_{s 0}
$$

where $\Gamma_{s}(x)$ is the transition matrix and $\xi_{s 0}$ the upstream condition at $x=0$.

To solve this equation, let us diagonalize matrix $\mathcal{A}_{s}$ :

$$
\mathcal{A}_{s}=P_{s} D_{s} P_{s}^{-1}
$$

with 


$$
\begin{aligned}
D_{s} & =\left(\begin{array}{cc}
\lambda_{1}(s) & 0 \\
0 & \lambda_{2}(s)
\end{array}\right), \\
P_{s} & =\frac{1}{T s}\left(\begin{array}{cc}
T s & T s \\
-\lambda_{1}(s) & -\lambda_{2}(s)
\end{array}\right), \\
P_{s}^{-1} & =\frac{1}{\lambda_{1}(s)-\lambda_{2}(s)}\left(\begin{array}{cc}
-\lambda_{2}(s) & -T s \\
\lambda_{1}(s) & T s
\end{array}\right),
\end{aligned}
$$

and the eigenvalues:

$$
\begin{aligned}
\lambda_{i} & =\alpha+(-1)^{i} \beta, i=1,2 \\
\alpha & =a+b s \\
\beta & =\sqrt{\left(2 a d+c^{2}\right) s^{2}+2 a c s+a^{2}}
\end{aligned}
$$

with $a=\frac{\nu}{2 T\left(\mathcal{C}^{2}-V^{2}\right)}, b=\frac{V}{\mathcal{C}^{2}-V^{2}}, c=\frac{V \nu-\left(\mathcal{C}^{2}-V^{2}\right) T \mu}{\nu\left(\mathcal{C}^{2}-V^{2}\right)}, d=\frac{1}{2 a}\left[\frac{\mathcal{C}^{2}}{\left(\mathcal{C}^{2}-V^{2}\right)^{2}}-c^{2}\right]$.

In the uniform case, the transition matrix $\Gamma_{s}(x)$ of (A.2) between 0 and $x$, is given by the following closed-form expression ([14]):

$$
\Gamma_{s}(x)=P_{s} e^{D_{s} x} P_{s}^{-1}=\left(\begin{array}{cc}
\frac{\lambda_{1} e^{\lambda_{2} x}-\lambda_{2} e^{\lambda_{1} x}}{\lambda_{1}-\lambda_{2}} & \frac{T s\left(e^{\lambda_{2} x}-e^{\lambda_{1} x}\right)}{\lambda_{1}-\lambda_{2}} \\
\frac{\lambda_{1} \lambda_{2}\left(e^{\lambda_{1} x}-e^{\lambda_{2} x}\right)}{T s\left(\lambda_{1}-\lambda_{2}\right)} & \frac{\lambda_{1} e^{\lambda_{1} x}-\lambda_{2} e^{\lambda_{2} x}}{\lambda_{1}-\lambda_{2}}
\end{array}\right)
$$

Finally the solution of the ordinary differential Eq. (A.2) is:

$$
\left(\begin{array}{l}
q(x, s) \\
y(x, s)
\end{array}\right)=\Gamma_{s}(x)\left(\begin{array}{l}
q(0, s) \\
y(0, s)
\end{array}\right)
$$

\section{B Taylor series expansion of the Saint-Venant transfer function}

The method to obtain the Taylor series expansion of the Saint-Venant transfer function is detailed in this section.

$$
T F(x, s)=A(x)+B(x) s+C(x) s^{2}+D(x) s^{3}+o\left(s^{3}\right)
$$




\section{B.1 Some preliminary computations}

The elements of the transfer matrix $\Gamma$ are expressed as functions of $\lambda_{1}$ and $\lambda_{2}$ or as functions of $\alpha$ and $\beta$. So writing the Taylor series of $\alpha$ and $\beta$ leads to the Taylor series of the elements of $\Gamma(x, s)$.

To make sure that no term will be forgotten in the computations, we choose to compute the fourth order Taylor series of $\alpha$ and $\beta$, given by:

$$
\begin{aligned}
& \alpha(s)=a+b s \\
& \beta(s)=a+c s+d s^{2}-\frac{c d}{a} s^{3}+\left(\frac{c^{2} d}{a^{2}}-\frac{d^{2}}{2 a}\right) s^{4}
\end{aligned}
$$

In order to simplify the computations, we define three intermediate variables $Z_{1 x}(s)$, $Z_{2 x}(s), Z_{3 x}(s)$. The following equations resume these variables and their Taylor series:

$$
Z_{1 x}(s)=e^{-2 \beta x}=A_{1 x}+B_{1 x} s+C_{1 x} s^{2}+D_{1 x} s^{3}+E_{1 x} s^{4}
$$

$$
\begin{aligned}
A_{1 x} & =e^{-2 a x} \\
B_{1 x} & =-2 c x e^{-2 a x} \\
C_{1 x} & =-2 x\left(d-c^{2} x\right) e^{-2 a x} \\
D_{1 x} & =-4 c x\left[\frac{1}{3} c^{2} x^{2}-2 a d\left(1+\frac{x}{2 a}\right)\right] e^{-2 a x} \\
E_{1 x} & =\left[\left(\frac{d^{2} x}{a}-2 \frac{c^{2} d x}{a^{2}}\right)(1+2 a x)-4 c^{2} d x^{3}+\frac{2}{3} c^{4} x^{4}\right] e^{-2 a x} \\
& Z_{2 x}(s)=e^{(\alpha+\beta) x}=A_{2 x}+B_{2 x} s+C_{2 x} s^{2}+D_{2 x} s^{3}+E_{2 x} s^{4}
\end{aligned}
$$

$$
\begin{aligned}
A_{2 x} & =e^{2 a x} \\
B_{2 x} & =(b+c) x e^{2 a x} \\
C_{2 x} & =\left(d x+\frac{1}{2}(b+c)^{2} x^{2}\right) e^{2 a x} \\
D_{2 x} & =\left(-\frac{c d x}{a}+(b+c) d x^{2}+\frac{1}{6}(b+c)^{3} x^{3}\right) e^{2 a x} \\
E_{2 x} & =\left[\frac{c^{2} d x}{a^{2}}-\frac{c d x^{2}}{a}(b+c)-\frac{d^{2} x}{2 a}(1-a x)+\frac{1}{2} x^{2}(b+c)^{2}\left(d x+\frac{1}{12}(b+c)^{2} x^{2}\right)\right] e^{2 a x} \\
& Z_{3 x}(s)=\frac{\alpha^{2}-\beta^{2}}{T s}=A_{3 x}+B_{3 x} s+C_{3 x} s^{2}+D_{3 x} s^{3}+E_{3 x} s^{4}
\end{aligned}
$$




$$
\begin{aligned}
A_{3 x} & =\frac{2}{T} a(b-c) \\
B_{3 x} & =\frac{1}{T}\left(b^{2}-c^{2}-2 a d\right) \\
C_{3 x} & =0 \\
D_{3 x} & =0 \\
E_{3 x} & =0
\end{aligned}
$$

We also define two operators $\mathcal{M}$ and $\mathcal{D}$. These operators are used to compute the Taylor series of the multiplication $F_{1}(s) F_{2}(s)$ and the division $F_{1}(s) / F_{2}(s)$, where $F_{1}(s)=A_{1}+B_{1} s+C_{1} s^{2}+D_{1} s^{3}+E_{1} s^{4}$ and $F_{2}(s)=A_{2}+B_{2} s+C_{2} s^{2}+D_{2} s^{3}+E_{2} s^{4}$.

If $F_{M}=\mathcal{M}\left(F_{1}, F_{2}\right)$ and $F_{D}=\mathcal{D}\left(F_{1}, F_{2}\right)$ and if the Taylor series of $F_{M}(s)$ and $F_{D}(s)$ are given by:

$$
\begin{gathered}
F_{M}(s)=A_{M}+B_{M} s+C_{M} s^{2}+D_{M} s^{3}+E_{M} s^{4} \\
F_{D}(s)=A_{D}+B_{D} s+C_{D} s^{2}+D_{D} s^{3}+E_{D} s^{4}
\end{gathered}
$$

then operators $\mathcal{M}$ and $\mathcal{D}$ give:

$$
\begin{aligned}
& A_{M}=A_{1} A_{2} \\
& B_{M}=A_{1} B_{2}+B_{1} A_{2} \\
& C_{M}=A_{1} C_{2}+B_{1} B_{2}+C_{1} A_{2} \\
& D_{M}=A_{1} D_{2}+B_{1} C_{2}+C_{1} B_{2}+D_{1} A_{2} \\
& E_{M}=A_{1} E_{2}+B_{1} D_{2}+C_{1} C_{2}+D_{1} B_{2}+E_{1} A_{2}
\end{aligned}
$$

and

$$
\begin{aligned}
A_{D}= & \frac{A_{1}}{A_{2}} \\
B_{D}= & \frac{1}{A_{2}}\left(B_{1}-\frac{A_{1} B_{2}}{A_{2}}\right) \\
C_{D}= & \frac{1}{A_{2}}\left[C_{1}-\frac{B_{1} B_{2}}{A_{2}}-\frac{A_{1}}{A_{2}}\left(C_{2}-\frac{B_{2}^{2}}{A_{2}}\right)\right] \\
D_{D}= & \frac{1}{A_{2}}\left[D_{1}-\frac{C_{1} B_{2}}{A_{2}}-\frac{B_{1}}{A_{2}}\left(C_{2}-\frac{B_{2}^{2}}{A_{2}}\right)-\frac{A_{1}}{A_{2}}\left(D_{2}-2 \frac{B_{2} C_{2}}{A_{2}}+\frac{B_{2}^{3}}{A_{2}^{2}}\right)\right] \\
E_{D}= & \frac{1}{A_{2}}\left[E_{1}-\frac{D_{1} B_{2}}{A_{2}}-\frac{C_{1}}{A_{2}}\left(C_{2}-\frac{B_{2}^{2}}{A_{2}}\right)-\frac{B_{1}}{A_{2}}\left(D_{2}-2 \frac{B_{2} C_{2}}{A_{2}}+\frac{B_{2}^{3}}{A_{2}^{2}}\right)\right. \\
& \left.-\frac{A_{1}}{A_{2}}\left(E_{2}-2 \frac{B_{2} D_{2}+C_{2}^{2}}{A_{2}}+3 \frac{B_{2}^{2} C_{2}}{A_{2}^{2}}-\frac{B_{2}^{4}}{A_{2}^{3}}\right)\right]
\end{aligned}
$$

We define a last intermediate variable $\delta_{x}(s)$ as following:

$$
\delta_{x}(s)=\frac{1-e^{-2 \beta x}}{2 \beta} e^{(\alpha+\beta) x}=\frac{1-Z_{1 x}}{2 \beta} Z_{2 x}
$$


Knowing the Taylor series of $Z_{1 x}$ and $Z_{2 x}$ and using the two operators $\mathcal{M}$ and $\mathcal{D}$, we can compute the Taylor series of the function $\delta_{x}(s)$ :

$$
\delta_{x}(s)=\mathcal{M}\left[\mathcal{D}\left(1-Z_{1 x}, 2 \beta\right), Z_{2 x}\right]
$$

\section{B.2 Transfer Matrix $\Gamma$ and Transfer Function $T F$}

All the elements of the transfer matrix $\Gamma(x, s)$ can be expressed from the four variables previously introduced.

$$
\begin{aligned}
& \gamma_{11}(x, s)=Z_{2 x}(s)-(\alpha+\beta) \delta_{x}(s) \\
& \gamma_{12}(x, s)=-T s \delta_{x}(s) \\
& \gamma_{21}(x, s)=Z_{3 x}(s) \delta_{x}(s) \\
& \gamma_{22}(x, s)=Z_{2 x}(s)+(\alpha-\beta) \delta_{x}(s)
\end{aligned}
$$

So knowing the Taylor series of these intermediate variables, we can use the two operators $\mathcal{M}$ and $\mathcal{D}$ to compute the Taylor series of each $\gamma_{i j}(x, s)$.

$$
\begin{aligned}
& \gamma_{11}(x, s)=Z_{2 x}(s)-\mathcal{M}\left(\alpha+\beta, \delta_{x}(s)\right) \\
& \gamma_{12}(x, s)=-\mathcal{M}\left(T s, \delta_{x}(s)\right) \\
& \gamma_{21}(x, s)=\mathcal{M}\left(Z_{3 x}(s), \delta_{x}(s)\right) \\
& \gamma_{22}(x, s)=Z_{2 x}(s)+\mathcal{M}\left(\alpha-\beta, \delta_{x}(s)\right)
\end{aligned}
$$

In the same way, we compute the Taylor series of $\gamma_{i j}(X, s)$.

Then it is possible to compute the Taylor series of $k_{0}(X, s)$ (Eq. (14)):

$$
k_{0}(X, s)=-\mathcal{D}\left(\gamma_{12}(X, s)-k \gamma_{22}(X, s), \gamma_{11}(X, s)-k \gamma_{21}(X, s)\right) .
$$

Finally we get the Taylor series of $T F(x, s)$ (Eq. (15)):

$$
T F(x, s)=\gamma_{11}(x, s)+\mathcal{D}\left(\gamma_{12}(x, s), k_{0}(X, s)\right)
$$

One can remark that for $s=0, \gamma_{11}(x, 0)=1$ and $\gamma_{12}(x, 0)=0$, which leads to $T F(x, 0)=A(x)=1$. This means that the static gain of the approximate transfer function is equal to 1 . This result is in agreement with the conservative property of the flow. 


\section{Taylor series expansion of the Saint-Venant transfer function in the non uniform case}

In the non-uniform case, the reach is split into two sub-pools. Variables $a, b, c, d$, $x, X, T, \alpha, \beta$ are replaced by their values corresponding to each sub-pool. In that sense, matrix $\Gamma(x, s)$ is replaced by $\underline{\Gamma}(\underline{x}, s)$ and $\bar{\Gamma}(\bar{x}, s)$ respectively, where $\underline{x}=x$ and $\underline{X}=x_{1}$ are the relative position and the length of the uniform part, and $\bar{x}=x-x_{1}$ and $\bar{X}=X-x_{1}$ are the relative position and the length of the backwater part $\left(x_{1}\right.$ is the abscissa of the connection point).

The Taylor series of $\underline{\Gamma}(\underline{x}, s)$ and $\bar{\Gamma}(\bar{x}, s)$ are computed for each sub-pool (see appendix B). In the backwater part $\left(x_{1} \leq x \leq X\right)$, the Taylor series of $\hat{\Gamma}(x, s)$ is computed using operator $\mathcal{M}$.

Then we can compute the Taylor series of $\hat{k}_{0}(X, s)$ (Eq. (34)):

$$
\hat{k}_{0}(X, s)=-\mathcal{D}\left(\hat{\gamma}_{12}(X, s)-k \hat{\gamma}_{22}(X, s), \hat{\gamma}_{11}(X, s)-k \hat{\gamma}_{21}(X, s)\right) .
$$

Finally we get the Taylor series of $T F(x, s)$ (Eq. (33)):

$$
T F(x, s)=\hat{\gamma}_{11}(x, s)+\mathcal{D}\left(\hat{\gamma}_{12}(x, s), \hat{k}_{0}(X, s)\right)
$$

\section{Moment matching method}

\section{D.1 Second-order-with-delay}

The transfer function $T F$ is approximated by a second-order-with-delay:

$$
q(x, s)=\frac{g(x) e^{-\tau(x) s}}{\left(1+K_{1}(x) s\right)\left(1+K_{2}(x) s\right)} q(0, s)
$$

We use the following property of the $R$-th cumulant $M_{R}$ (logarithmic moment) of a function $h(s)$ expressed in the Laplace domain:

$$
M_{R}[h(s)]=(-1)^{R} \frac{d^{R}}{d s^{R}}[\log h(s)]
$$

The first four cumulants of $T F(x)$ are denoted $M_{0}(x), M_{1}(x), M_{2}(x)$ and $M_{3}(x)$. 
Equating the cumulants of $T F$ and its approximate form leads to:

$$
\left\{\begin{array}{l}
M_{0}(x)=\log g(x) \\
M_{1}(x)=\tau(x)+K_{1}(x)+K_{2}(x) \\
M_{2}(x)=K_{1}^{2}(x)+K_{2}^{2}(x) \\
M_{3}(x)=2 K_{1}^{3}(x)+2 K_{2}^{3}(x)
\end{array}\right.
$$

Let $S=K_{1}+K_{2}$ and $P=K_{1} K_{2}$ :

$$
\begin{aligned}
& S^{2}=M_{2}+2 P \\
& S^{3}=\frac{M_{3}}{2}+3 P S
\end{aligned}
$$

which leads to the third order equation:

$$
S^{3}-3 M_{2} S+M_{3}=0
$$

We can find $(u, v)$ such as $u+v=S$ and $u v=T$. Then (D.6) leads to:

$$
u^{3}+v^{3}+3\left(T-M_{2}\right)(u+v)+M_{3}=0
$$

If we choose $T=M_{2}$, we obtain the following system:

$$
\begin{aligned}
u^{3}+v^{3} & =-M_{3} \\
u^{3} v^{3} & =M_{2}^{3}
\end{aligned}
$$

$u^{3}$ and $v^{3}$ are solutions of the second order equation:

$$
X^{2}+M_{3} X+M_{2}^{3}=0
$$

If $M_{3}^{2} \leq 4 M_{2}^{3}$, the solutions of (D.10) are complex and this ensure the stability of the approximation by a second-order-with-delay. Otherwise the Saint-Venant transfer function can be approximated by a first-order-with-delay. The system to be solved is then given by the first three equations of the system (D.3) with $K_{2}=0$. 
In the case where $M_{3}^{2}<4 M_{2}^{3}$, the solution of (D.3) is given by:

$$
\left\{\begin{aligned}
\phi & =\frac{\pi}{2}+\arctan \left(\frac{M_{3}}{\sqrt{4 M_{2}^{3}-M_{3}^{2}}}\right) \\
S & =2 \sqrt{M_{2}} \cos \frac{\phi}{3} \\
P & =-M_{2}-\frac{M_{3}}{2 S} \\
\tau & =M_{1}-S \\
K_{1} & =\frac{S+\sqrt{S^{2}-4 P}}{2} \\
K_{2} & =\frac{S-\sqrt{S^{2}-4 P}}{2} \\
g & =1
\end{aligned}\right.
$$

\section{D.2 First-order-with-delay}

In the case where the second order approximate model is unstable $\left(M_{3}^{2} \geq 4 M_{2}^{3}\right)$, it is possible to replace the second order by a first order.

$$
q(x, s)=\frac{g(x) e^{-\tau(x) s}}{1+K_{1}(x) s} q(0, s)
$$

Then $K_{2}=0$, and $\tau$ and $K_{1}$ are determined by equating the first three cumulants of the exact transfer function and the approximate one:

$$
\left\{\begin{array}{l}
M_{0}(x)=\log g(x) \\
M_{1}(x)=\tau(x)+K_{1}(x) \\
M_{2}(x)=K_{1}^{2}(x)
\end{array}\right.
$$

which leads to:

$$
\left\{\begin{aligned}
\tau(x) & =M_{1}(x)-\sqrt{M_{2}(x)} \\
K_{1}(x) & =\sqrt{M_{2}(x)} \\
g(x) & =1
\end{aligned}\right.
$$

